\title{
Meglumine cyclic adenylate improves neurological function following acute spinal cord injury in rats
}

\author{
JINGWU LIAO ${ }^{1}$, JINGMING XIE ${ }^{1}$, DAQIANG LIN ${ }^{2}$, NING LU $^{1}$, LIMIN GUO ${ }^{1}$, WEIQIANG LI ${ }^{1}$, \\ BO PU ${ }^{1}$, YANG YANG ${ }^{1}$, ZHENLONG YANG $^{1}$, YING ZHANG $^{1}$ and YUEMING SONG ${ }^{3}$ \\ ${ }^{1}$ Department of Orthopaedic Surgery, The 2nd Affiliated Hospital of Kunming Medical University, Kunming, Yunnan 650031;
${ }^{2}$ Department of Orthopaedic Surgery, Hospital of Integrated Traditional and Western Medicine, Dazhou, Sichuan 635000;
${ }^{3}$ Department of Orthopaedic Surgery, West China Hospital, Sichuan University, Chengdu, Sichuan 610041, P.R. China
}

Received November 15, 2013; Accepted May 14, 2014

DOI: $10.3892 / \mathrm{mmr} .2014 .2352$

\begin{abstract}
Elevation of intracellular cyclic adenosine monophosphate (cAMP) levels facilitates recovery following spinal injury by suppressing secondary pathology and promoting axonal regeneration. However, this treatment strategy is limited by lack of effective and tolerable clinical agents. The present study examined the effects of meglumine cyclic adenylate (MCA) on neurological recovery, cAMP concentration, adenylate cyclase 3 (AC3) activity and phosphodiesterase 4D (PDE4D) activity during early stage acute spinal cord injury (SCI) in rats. A total of 48 Sprague-Dawley rats were randomly assigned to groups $\mathrm{A}, \mathrm{B}$ or $\mathrm{C}$, each consisting of 16 animals. SCI was induced by Allen's method using a $7 \mathrm{~g} \mathrm{x} 3 \mathrm{~cm}$ extradural weight-drop impact on spinal cord segment T11. A total of 30 min following SCI, group A received a single $30 \mathrm{mg} / \mathrm{kg}$-bw i.p. dose of methylprednisolone, group B received $2 \mathrm{mg} / \mathrm{kg}$-bw i.p. MCA daily for seven days and group $\mathrm{C}$ were administered an equal volume of normal saline. Seven days following SCI, the spinal cord samples from eight rats per group were obtained to measure the cAMP concentration, and the activities of AC3 and PDE4D. The remaining eight rats per group were used for behavioral assessments using the inclined plane stability test and Gale scale for up to six weeks post-SCI. The drug-treated groups A and B had higher cAMP concentrations and AC3 activities but lower PDE4D activities at the lesion sites, as well as superior behavioral scores post-SCI compared with the vehicle-treated group $\mathrm{C}(\mathrm{P}<0.05)$. Furthermore, cAMP was higher in group B than in group A $(\mathrm{P}<0.05)$. It was concluded that MCA may serve as an effective $\mathrm{SCI}$ treatment by activating $\mathrm{AC} 3$ and suppressing PDE4D.
\end{abstract}

Correspondence to: Dr Yueming Song, Department of Orthopaedic Surgery, West China Hospital, Sichuan University, No. 37 Guo Xue Xiang, Chengdu, Sichuan 610041, P.R. China E-mail:sym_hx@163.com

Key words: meglumine cyclic adenylate, spinal cord injury, cyclic adenosine monophosphate, metabolism phosphodiesterase 4D, adenylate cyclase 3

\section{Introduction}

Spinal cord injury (SCI) is characterized by an immediate, irreversible destruction of tissue at the lesion site with secondary expansion of damage over time, resulting in irreversible loss of sensation and movement. The consequences of SCI are lifetime debilitation, the extent of which increases with more rostral injury site (1). Global estimates of the number of new cases annually range from 15 40/million (2). There is currently no effective treatment for acute SCI, except for high-dose methylprednisolone (MP) therapy, the efficacy of which has been challenged by numerous studies, due to the marginal therapeutic effects and high incidence of pulmonary complications $(3,4)$. An ideal post-SCI pharmacotherapy would suppress the pathogenic processes leading to secondary injury, including inflammation and apoptosis, and promote nerve regeneration $(5,6)$.

Numerous studies have demonstrated a significant decrease in neuronal cyclic adenosine monophosphate (cAMP) following SCI, which may exacerbate secondary neuronal injury and inhibit neural regeneration $(7,8)$. Therefore, elevation of cAMP in neurons may facilitate recovery or reduce secondary pathology following SCI. Indeed, PDE4 inhibitors have been implicated as possible therapeutics to reduce secondary pathological responses and activate regenerative mechanisms in animal models of SCI, by maintaining cAMP levels $(9,10)$. The mechanisms for the post-SCI decrease in cAMP are elusive, and a lack of selective agents with tolerable side effects has hampered the clinical application of this treatment strategy. cAMP is a ubiquitous secondary messenger in all life forms. In mammals, intracellular levels are under the dual regulation of adenylate cyclases (ACs), which are activated by numerous different receptors and by phosphodiesterases (PDEs), and are also under complex upstream regulation (11). There are ten known $\mathrm{AC}$ isoforms (AC1-10), each with distinct modes of regulation and expression patterns (12) and four different cAMP-specific PDE4 isozymes (PDE4A, PDE4B, PDE4C and PDE4D) (13). Several studies have indicated that PDE4D is expressed by spinal cord oligodendrocytes (14). The AC3 isoform is distributed throughout the brain, spinal cord and olfactory epithelium $(15,16)$. The present study examined the possibility that SCI triggers a post-traumatic inhibition 
of $\mathrm{AC}$ and (or) activation of PDE, thereby causing a decline in cellular cAMP concentration, and whether agents that maintain post-injury intracellular cAMP concentration may limit secondary damage and improve functional outcome. Meglumine cyclic adenylate (MCA) is a cAMP analog currently available for cardiovascular treatments in China. This compound of cAMP and meglumine acts both as a cAMP analogue and a PDE inhibitor, and may therefore be a particularly efficient cAMP upregulator. In the present study, the post-SCI changes in AC3 and PDE4D activities, tissue cAMP concentrations and the recovery of motoric function were examined in a rat model of SCI.

\section{Materials and methods}

Animals and surgical methods. A total of 48 healthy adult Sprague-Dawley (SD) rats of (age, 6-8 months) both sexes (Laboratory Animal Center of Sichuan University, Chengdu, China) weighing 250-310 g (mean, $280 \mathrm{~g}$ ) were used. The animals were maintained in standard housing conditions $\left(22 \pm 2^{\circ} \mathrm{C}, 55 \%\right.$ humidity, light from 6:00 am to 8:00 pm) and fed a standard dry diet and water ad libitum. All of the experimental procedures were approved by the Institutional Animal Care and Use Committee of Sichuan University. Following experimental SCI, the rats were randomly divided into three equal treatment groups of 16: A (methylprednisolone group), B (MCA group) and C (control group). An acute T11 SCI was induced by Allen's method under aseptic conditions (17). Briefly, under $1 \%$ pentobarbital sodium (30 mg/kg-ip) anesthesia, the rats were fixed in a prone position on a small arched table, and the hair at the operation site (around T11) was shaved and treated with povidone iodine solution. One to three minutes prior to the surgical procedure, the rats were administered $2.5 \mathrm{ml}$ of warm saline solution containing penicillin $\mathrm{G}$ sodium (10,000 units/kg-bw) by subcutaneous injection in the abdominal wall as an antimicrobial agent. A $3-\mathrm{cm}$ skin incision was made posterior to the midline and continued down to the lumbosacral fascia, which was incised to expose the tips of the spinous processes. With blunt dissection, the paraspinal musculature was subperiosteally dissected and the lumbar vertebral segments exposed. A T10-T12 total laminectomy was performed to expose the spinal dura mater and nerve roots. A small, curved, hard and sterilized plastic plate was placed on the surface of the T11 dura mater, and an SCI was induced by dropping a 7-g weight from $3 \mathrm{~cm}$ onto the plate. Upon completion of the surgical procedure, the lumbosacral fascia and other layers were sutured layer by layer. A total of $30 \mathrm{~min}$ following the injury, group A was intraperitoneally injected with a single $30 \mathrm{mg} / \mathrm{kg}$ bw dose of methylprednisolone (Pfizer/Pharmacia and Upjohn, Kalamazoo, MI, USA), group B with $2 \mathrm{mg} / \mathrm{kg}$ MCA (Wang Bang Biochemical Pharmaceutical Co., Ltd., China) once daily for seven days, and group $\mathrm{C}$ with an equal volume of saline. Following the surgical procedure, the animals also received $2.5 \mathrm{ml}$ warm saline solution containing penicillin $\mathrm{G}$ sodium (10,000 units $/ \mathrm{kg}$ bw). The animals were positioned over a heating pad to maintain their body temperature and administered a light concentration of diethyl ether ( $1 \mathrm{ml}$ diethyl ether in a $250-\mathrm{ml}$ wide-mouthed bottle for 0.5-1.0 min) for pain relief following waking. Following SCI, the rats were housed one per cage. Each underwent manual bladder evacuation three times daily and wound dressings were changed daily. Eight randomly selected animals from each group were examined for six weeks on the inclined plane and Gale scale tests. Seven days following SCI, the spinal cord samples were obtained from the other eight rats in each group for pathological examination and to determine the cAMP levels and both AC3 and PDE4D activities by a specific ELISA and quantitative immunohistochemistry.

\section{Behavioral testing}

Inclined plane. The inclined plane test assesses the ability of an animal to maintain position on a slope. The highest angle of inclination maintained for $5 \mathrm{sec}$ was determined as a measure of stability. The test was conducted from week one to week six post-SCI (18). Functional motor deficits and recovery are expressed by the inclined plane (IP) maintenance ratio (post-injury/pre-injury).

Gale scale. The animals' motor functions were also observed and scored into six levels by the Gale scale (Table I) (19).

\section{Pathological examination}

Tissue sample preparation. Seven days following injury, half of the animals in groups A-C were anesthetized by ether inhalation and the injured spinal cord was re-exposed. The entire section of injured spinal column was excised by breaking the upper, lower, left and right vertebrae connected to the ribs. A $1.5-\mathrm{cm}$ section of the vertebral column containing the lesion site was cut into two equal portions through the center of the lesion site. The upper-half portion (rostral side, from the injury epicenter tissue up to $6 \mathrm{~mm}$ rostral side) was fixed in $10 \%$ neutral formalin for $12 \mathrm{~h}$, and then the spinal cord was isolated and further fixed for another $12 \mathrm{~h}$ for pathological examination. The remaining caudal portion (caudal side, from the injury epicenter tissue up to $9 \mathrm{~mm}$ caudal side) was further cut into three equal portions, which were immediately wrapped in aluminum foil and immersed in liquid nitrogen for later cAMP assay and estimation of AC3 and PDE4D activities. The upper portion of the injured spinal cord sample (rostral side) was assessed for cAMP by ELISA, the middle portion was used to detect AC3, and the lower portion (caudal side) was used to detect PDE4D by immunohistochemistry.

Hematoxylin and eosin $(H \& E)$ staining. The tissue samples were fixed in $10 \%$ neutral formalin for $24 \mathrm{~h}$, dehydrated, embedded in paraffin and sectioned into 5- $\mu \mathrm{m}$ slices. The middle sections were treated with H\&E. An Olympus optical microscope CX21 was used (Olympus, Tokyo, Japan).

Measurement of cAMP. The cAMP content was measured by ELISA (2nd Generation, DE0450; R\&D Inc., R\&D Systems, Inc., Minneapolis, MN, USA). Spinal cord samples were removed from the liquid nitrogen, ground, weighed and dissolved in ten volumes of 5\% trichloroacetic acid (TCA). After the raw lysate was centrifuged at $40 \mathrm{~g}$ for $10 \mathrm{~min}$, the supernatant was drawn and mixed with three volumes of hydrated ether. Following dehydration, the ED2 detection buffer was added. A 200- $\mu$ l sample was mixed with $10 \mu 1$ acetic anhydride reagent. Similarly, $1 \mathrm{ml}$ cAMP standard in ED2 detection phosphate-buffered saline was mixed with 
Table I. Effects of MP (group A) and MCA (group B) on motor function six weeks following spinal cord injury, compared with the saline-treated controls (group C).

\begin{tabular}{lccc}
\hline Groups & $\mathrm{N}$ & $\begin{array}{c}\text { Gale scale } \\
\text { (point) }\end{array}$ & $\begin{array}{c}\text { IP maintaining ratio } \\
(\%)\end{array}$ \\
\hline A (MP) & 8 & $3.56 \pm 0.26$ & $0.39 \pm 0.023$ \\
B (MCA) & 8 & $3.67 \pm 0.29$ & $0.42 \pm 0.021$ \\
C (saline) & 8 & $2.68 \pm 0.21^{\mathrm{a}}$ & $0.28 \pm 0.012^{\mathrm{a}}$ \\
P-value & & $<0.05$ & $<0.05$
\end{tabular}

${ }^{a} \mathrm{P}<0.05$ compared with groups $\mathrm{A}$ and $\mathrm{B}$; data are expressed as the mean \pm standard deviation. MP, methylprednisolone; MCA, meglumine cyclic adenylate; IP, inclined plane.
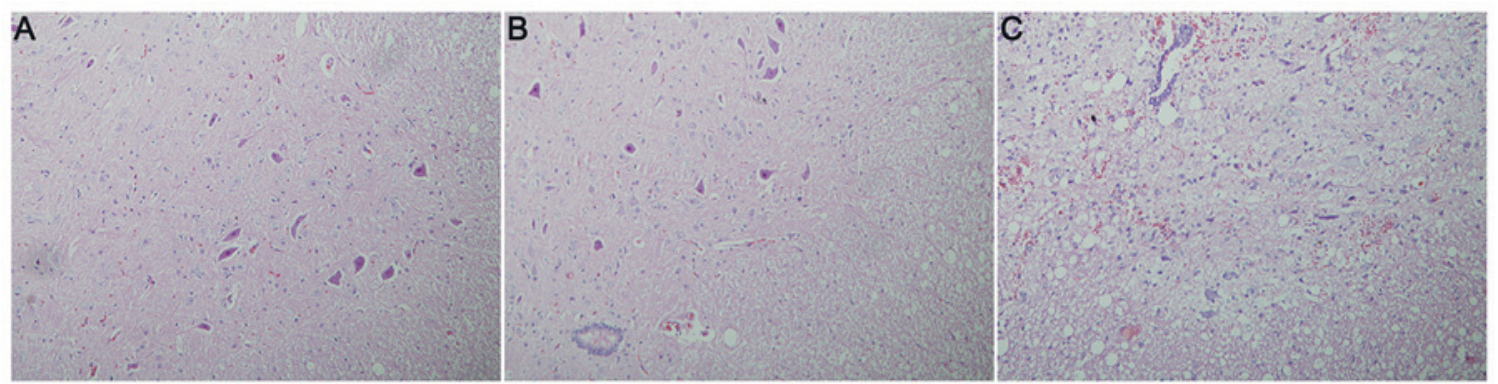

Figure 1. (A) H\&E staining of spinal cord at the injury site in (A) methylprednisolone-treated rats in group A, (B) meglumine cyclic adenylate-treated rats in group B and (C) saline-treated rats in group C (magnification, $\mathrm{x} 200$ ). H\&E, hematoxylin and eosin.

$50 \mu 1$ acetylation reagent. The cAMP conjugate, cAMP antibody solution, $p$-nitrophenyl phosphatase substrate and $50 \mu 1$ of the reaction termination solution were added to each micropore. An ELISA reader (detection wavelength, $405 \mathrm{~nm}$ ) was used to rapidly determine the optical density (OD) of each micropore. A standard curve was plotted using the logarithmic concentration of acetylated standard cAMP (x-axis) vs. the OD value (y-axis). The cAMP concentrations of the samples were estimated from the standard curve.

Estimation of AC3 and PDE4D activity by immunohistochemistry. Cryostat sections were cut at 4-8 $\mathrm{mm}$, warmed to room temperature (30 $\mathrm{min}$ ) and fixed in acetone for $10 \mathrm{~min}$ at $4^{\circ} \mathrm{C}$. Fixed slices were washed three times in phosphate-buffered saline (PBS; $5 \mathrm{~min} /$ wash), soaked in 3\% hydrogen peroxide for 5-10 min to quench endogenous peroxidase activity, and then washed again in PBS (5 min x 3). To determine the number of AC3- and PDE4D-positive cells, the slices were incubated in rabbit polyclonal Santa's AC3 immunoglobulin (Ig)G or PDE4D IgG $(200 \mu \mathrm{g} / \mathrm{ml})$, followed by a secondary antibody (Beijing Zhongshan Golden Bridge Biotechnology Co., Ltd., Beijing, China) and visualization using a peroxidase-labeled streptavidin-avidin kit (Beijing Zhongshan Golden Bridge Biotechnology Co., Ltd.) and diaminobenzidine. Six arbitrary fields of view were chosen at high magnification (x400) for each AC3- or PDE4D-stained slice and six arbitrary neurons in each field were used to determine the average density of AC3 or PDE4D immunostaining in the cytoplasm. The mean density was used as an estimate of the AC3 or PDE4D activity.
Statistical analysis. The SPSS 12.0 software program (SPSS, Inc., Chicago, IL, USA) was used for statistical analyses. Values are presented as the mean \pm standard deviation. Group mean values were compared by one-way analysis of variance with post-hoc least-significant difference pair-wise comparisons (q-test).

\section{Results}

Behavioral assessments. Six weeks following SCI, the IP maintenance ratio and the Gale scale were significantly higher in treatment groups A (methylprednisolone) and B (MCA) compared with the vehicle-treated group $\mathrm{C}(\mathrm{P}<0.05)$, indicating improved recovery of motor function. Methylprednisolone and MCA were equally effective, as there was no significant difference in the behavioral assessments between groups A and B $(\mathrm{P}>0.05$; Table I).

\section{Pathological examination}

$H \& E$ staining. Higher densities of intact neurons were observed in groups A and B compared with those in group C. In spinal cord slices from drug-treated rats, the intercellular matrix was uniform, the majority of the neurons were morphologically normal, large and irregular, the membrane was intact, the cytoplasm was uniformly and lightly stained, and the nuclei and nucleoli were darkly stained and clear. Large numbers of small glial cells were observed among the neurons and there was little hemorrhage or edema (Fig. 1A and $\mathrm{B}$ ). In the control group (group C), however, the spinal cord tissues were sparse and significantly swollen with evident 
Table II. Mean density of AC3- and PDE4D-positive spinal neurons at the lesion site one week following spinal cord injury (mean \pm standard deviation).

\begin{tabular}{lccc}
\hline Groups & N & AC3-positive cell count & PDE4D-positive cell count \\
\hline A (MP) & 8 & $636.3 \pm 28.6$ & $239.3 \pm 21.7$ \\
B (MCA) & 8 & $622.2 \pm 36.1$ & $251.2 \pm 32.3$ \\
C (control group) & 8 & $372.8 \pm 33.2^{\mathrm{a}}$ & $568.1 \pm 39.2^{\mathrm{a}}$ \\
P-value & & $<0.05$ & $<0.05$ \\
\hline
\end{tabular}

${ }^{\text {aP }}<0.05$ compared with groups A and B. AC3, adenylate cyclase 3; PDE4D, phosphodiesterase 4D; MP, methylprednisolone; MCA, meglumine cyclic adenylate.
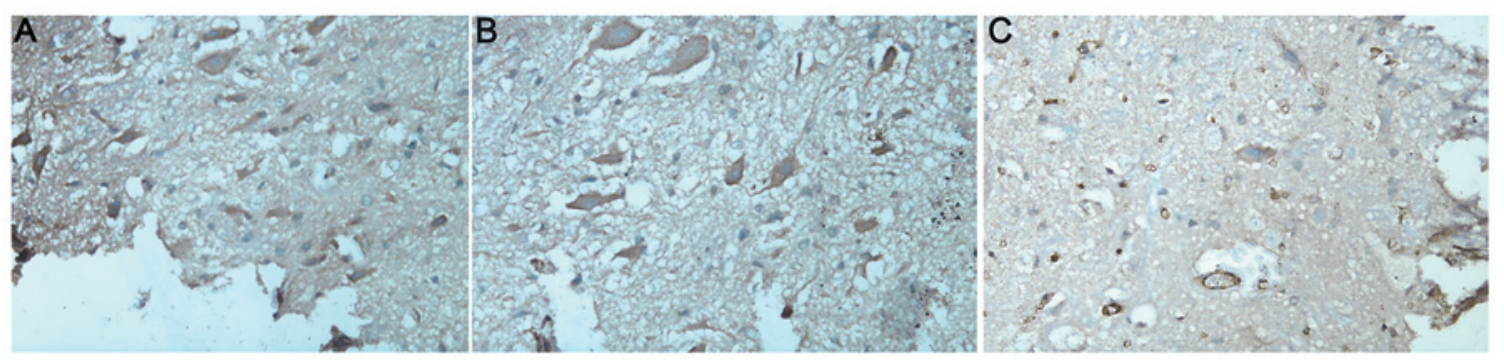

Figure 2. AC3 activity in the injured spinal cord of rats in groups (A) A, (B) B and (C) C as estimated by immunohistochemistry (magnification, x400). AC3, adenylate cyclase 3 .
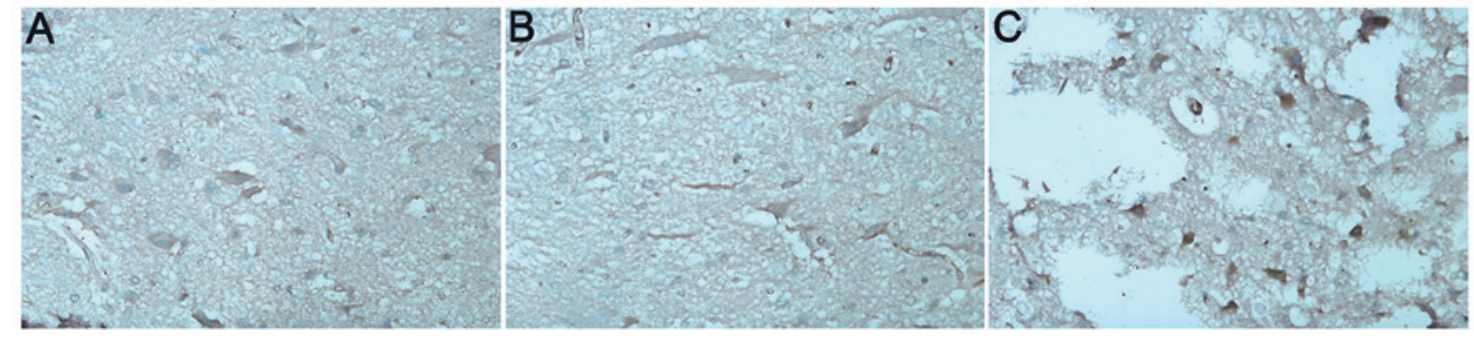

Figure 3. PDE4D activity in the injured spinal cord of rats in groups (A) A, (B) B and (C) C as estimated by immunohistochemistry (magnification, $\mathrm{x} 400$ ). PDE4D, phosphodiesterase 4D.

hemorrhage, few neurons were observed, and those remaining had swollen cell bodies, with uneven cytoplasmic staining. Numerous nuclei were contracted and degenerated (Fig. 1C).

AC3 activity. In groups $\mathrm{A}$ and $\mathrm{B}$, the neurons in the spinal cord were large and irregular with intact membranes. The nuclei and nucleoli were evident. Brown-yellow, dense and uniform cytoplasm immunostained for AC3 was visualized surrounding the nuclei (Fig. 2A and B). In the control group (group C), there were fewer neurons and those remaining had inhomogeneous cytoplasm with evident vacuolization and pale amber-yellow staining (Fig. 2C). The mean density of AC3-positive cells was lower than that in groups $\mathrm{A}$ and $\mathrm{B}(\mathrm{P}<0.05$; Table II).

PDE4D activity. In groups $\mathrm{A}$ and $\mathrm{B}$, the spinal neurons were large, irregularly shaped and exhibited intact membranes with clearly visible nuclei and nucleoli. These neurons were stained light yellow (Figs. 3A and B). In the control group (group C), there were fewer neurons, with inhomogeneous cytoplasm and evident vacuolations. These neurons were stained an amber color (Fig. 3C). The number of PDE-4D-positive cells (calculated as the mean density) was significantly higher than that in groups $\mathrm{A}$ and $\mathrm{B}(\mathrm{P}<0.05$; Table II).

cAMP concentration. Spinal cAMP levels at seven days following injury was higher in group A and B than those in group $\mathrm{C}(\mathrm{P}<0.05)$. Furthermore, cAMP levels in group $\mathrm{B}$ were higher those that in group $\mathrm{A}(\mathrm{P}<0.05$; Table III).

\section{Discussion}

cAMP and SPI. Cyclic adenosine monophosphate (cAMP) was first isolated from liver homogenates by Sutherland in 1957, and is now known to be distributed in all mammalian tissues except red blood cells (20). The concentration of cAMP is regulated by ACs and PDEs. The synthesis and degradation of cAMP is notably faster in nerve tissue than in other tissues, suggesting it has specific functions in rapid localized 
Table III. cAMP concentration at the lesion site seven days folllowing spinal cord injury.

cAMP concentration ( $\mathrm{pmol} / \mathrm{ml}$, mean \pm

\begin{tabular}{lcc} 
Groups & $\mathrm{N}$ & standard deviation) \\
\hline A (MP) & 8 & $70.0 \pm 3.6$ \\
B (MCA) & 8 & $112.5 \pm 4.5$ \\
C (control group) & 8 & $35.0 \pm 1.8^{\mathrm{a}}$ \\
P-value & & $<0.05$ \\
\hline
\end{tabular}

${ }^{\mathrm{a}} \mathrm{P}<0.05$ compared with groups $\mathrm{A}$ and $\mathrm{B}$. cAMP, cyclic adenosine monophosphate; MP, methylprednisolone; MCA, meglumine cyclic adenylate; SD, standard deviation.

intracellular signaling (21). Recent studies have demonstrated that cAMP levels in the spinal cord decrease following SCI, while the elevation of intracellular cAMP may reduce secondary SCI and promote regeneration $(9,22)$. Intracellular cAMP levels may be increased and maintained by a peripheral conditioning lesion, administration of cell-permeant cAMP analogs such as dbcAMP, or by treatment with PDE4 inhibitors, including rolipram. While each of these methods has been demonstrated to suppress secondary pathological responses, reduce the inhibition of axonal regeneration, and activate regenerative processes, each has specific disadvantages for routine clinical use. Nikulina et al (23) found that rolipram delivered for two weeks together with embryonic spinal cord transplantation following C3/4 SCI promoted axonal growth into the transplant, attenuated astrogliosis, a glial proliferative and hypertrophic reaction considered to block axonal elongation, and, most importantly, improved functional recovery in Evans-Hooded rats. The authors suggested that rolipram promoted regeneration of spinal axons by elevating neuronal cAMP concentration (23). Pearse et al (24) found that cAMP levels decreased by $\sim 70 \%$ following thoracic contusion SCI and remained below baseline for at least two weeks post-SCI. Immediate subcutaneous injection of rolipram prevented this decrease in cAMP and the occurrence of secondary SCI damage. In addition, this treatment promoted the growth of nerve fibers when combined with Schwann cell transplantation and direct dbcAMP injection into the injured site (24).

Consistent with other studies, the present study demonstrated that cAMP levels in the spinal cord were significantly below baseline seven days following SCI, while MCA and MP enhanced cAMP levels post-SCI (MCA more than MP). The MCA and MP groups also demonstrated improved maintenance of histological structure, with significantly more intact neurons, less tissue hemorrhaging and edema, and fewer regions of low cell density or necrosis, as revealed by $\mathrm{H} \& \mathrm{E}$ staining. In addition, MCA- and MP-treated rats demonstrated superior motor recovery as assessed by the IP stability test and Gale scale test battery compared with the control group. Administration of MCA and MP at the early stages of injury reversed the decrease and sustained cAMP levels post-SCI, possibly accounting for enhanced recovery of motor function. However, MCA may present certain advantages over other analogues or PDE4D inhibitors. Rolipram is also a monoamine oxidase inhibitor, which, at doses causing PDE4 suppression, may induce nausea and vomiting (25). By contrast, MCA acts as a cAMP analogue and PDE4 antagonist, and may therefore be more effective at lower doses.

ACs, PDEs and SCI. There are 11 classes of PDE (PDE1-11) and a total of 30 isozymes (26), of which PDE4, 7 and 8 are highly specific for the hydrolysis of cAMP, PDE5, 6 and 9 are highly specific for the hydrolysis of cGMP, and PDE1, 2, 3 are able to hydrolyze both cAMP and cGMP (but PDE3 has greater activity on cAMP). These PDE isoenzymes are differentially distributed in tissues and organs, with PDE4 broadly expressed in the nervous system. There are four PDE4 isozymes, PDE4A, PDE4B, PDE4C and PDE4D, mainly distributed within inflammatory and nerve cells (27). Pérez-Torres et al (28) detected abundant PDE4 in human, monkey and rat brains by in situ hybridization and immunohistochemistry. Similarly, Lamontagne et al (29) found PDE4D was widely distributed in the central nervous system (CNS), with particularly strong activity in the nodose ganglion, area postrema, solitary tract nucleus and locus coeruleus. There are ten adenylate cyclase (AC) isozymes (AC1-10) (30), and several of them are involved in signal transduction in the CNS. Abaffy et al (2003) found strong AC1 and AC3 expression in the nuclei of rat sensory neurons (30). In the present study, the AC3 and PDE4D activities in the spinal cord following SCI were also measured. Both MCA and MP treatments significantly increased the post-SCI AC3-positive cell count and reduced the PDE4D-positive cell count, thereby maintaining post-traumatic cAMP levels, protecting spinal neurons and improving post-SCI recovery.

MCA treatment mechanism for acute SCI. MCA is a compound of cAMP and meglumine with a mean blood half-life of 60-150 min. It has been used in China for the treatment of cardiovascular diseases with low toxicity and cost (32). In addition, MCA has physiological effects similar to cAMP and PDE inhibitors but with enhanced liposolubility and membrane permeability. In clinical cardiovascular applications, MCA serves as a cardiotonic agent to treat bradyarrhythmias or heart failure with high stability, low toxicity and low cost, but has not yet been used for studies on SCI treatment. The typical MCA dosage in adults is $1-3 \mathrm{mg} / \mathrm{kg} / \mathrm{d}$, while a dose of up to $2.5-5 \mathrm{mg} / \mathrm{kg} / \mathrm{d}$ (iv.qd) in children has been reported to be safe, as its $\mathrm{LD}_{50}$ is $1996 \pm 149 \mathrm{mg} / \mathrm{kg}$-iv (33). In the present study, a dose of $2 \mathrm{mg} / \mathrm{kg}$ was used, based on other clinical applications and preliminary experiments by our group.

In conclusion, cAMP levels in the spinal cord decreased following SCI, and elevation of intracellular cAMP levels reduced secondary $\mathrm{SCI}$ and increased the regenerative capacity. Therefore, cAMP analogues or tolerable PDE inhibitors may benefit patients in the early stages post-SCI. MCA is a dual cAMP analogue and PDE4 inhibitor and may be a prototype for an alternative treatment strategy. Further studies are required to examine its pharmacokinetics and full spectrum of actions following SCI. 


\section{References}

1. Wiedemann C: Repair: CSI in SCI. Nat Rev Neurosci 10: 621, 2009.

2. Cripps RA, Lee BB, Wing P, et al: A global map for traumatic spinal cord injury epidemiology: towards a living data repository for injury prevention. Spinal Cord 49: 493-501, 2011.

3. Ito Y, Sugimoto Y, Tomioka M, Kai N and Tanaka M: Does high dose methylprednisolone sodium succinate really improve neurological status in patient with acute cervical cord injury?: a prospective study about neurological recovery and early complications. Spine (Phila Pa 1976) 34: 2121-2124, 2009.

4. Bracken M: Steroids for acute spinal cord injury. Cochrane Database Syst Rev 3: CD001046, 2002.

5. Baptiste DC and Fehlings MG: Emerging drugs for spinal cord injury. Expert Opin Emerg Drugs 13: 63-80, 2008.

6. Kwon BK, Hillyer J and Tetzlaff W: Translational research in spinal cord injury: a survey of opinion from the SCI community. J Neurotrauma 27: 21-33, 2010.

7. Abe $\mathrm{N}$ and Cavalli V: Nerve injury signaling. Curr Opin Neurobiol 18: 276-283, 2008.

8. Udina E, Ladak A, Furey M, et al: Rolipram-induced elevation of cAMP or chondroitinase ABC breakdown of inhibitory proteoglycans in the extracellular matrix promotes peripheral nerve regeneration. Exp Neurol 223: 143-152, 2010.

9. Hannila SS and Filbin MT: The role of cyclic AMP signaling in promoting axonal regeneration after spinal cord injury. Exp Neurol 209: 321-332, 2008.

10. Mekhail M, Almazan $G$ and Tabrizian $M$ Oligodendrocyte-protection and remyelination post-spinal cord injuries: a review. Prog Neurobiol 96: 322-339, 2012.

11. Gancedo JM: Biological roles of cAMP: variations on a theme in the different kingdoms of life. Biol Rev Camb Philos Soc 88: 645-668, 2013.

12. Seifert R, Lushington GH, Mou TC, Gille A and Sprang SR Inhibitors of membranous adenylyl cyclases. Trends Pharmaco Sci 33: 64-78, 2012.

13. Michalski JM, Golden G, Ikari J and Rennard SI: PDE4: a nove target in the treatment of chronic obstructive pulmonary disease. Clin Pharmacol Ther 91: 134-142, 2012.

14. Whitaker CM, Beaumont E, Wells MJ, et al: Rolipram attenuates acute oligodendrocyte death in the adult rat ventrolateral funiculus following contusive cervical spinal cord injury. Neurosci Lett 438: 200-204, 2008.

15. Pavan B, Paganetto G and Dalpiaz A: Dopamine-sensitive adenylyl cyclases in neuronal development: physiopathological and pharmacological implications. Drug Discov Today 16 : 520-529, 2011.

16. Col JA, Matsuo T, Storm DR and Rodriguez I: Adenylyl cyclase-dependent axonal targeting in the olfactory system. Development 134: 2481-2489, 2007.

17. Koozekanani SH, Vise WM, Hashemi RM and McGhee RB: Possible mechanisms for observed pathophysiological variability in experimental spinal cord injury by the method of Allen. J Neurosurg 44: 429-434, 1976.
18. Rivlin AS and Tator $\mathrm{CH}$ : Objective clinical assessment of motor function after experimental spinal cord injury in the rat. J Neurosurg 47: 577-581, 1977.

19. Gale K, Kerasidis H and Wrathall JR: Spinal cord contusion in the rat: behavioral analysis of functional neurologic impairment. Exp Neurol 88: 123-134, 1985.

20. Gilman AG: Silver spoons and other personal reflections. Annu Rev Pharmacol Toxicol 52: 1-19, 2012.

21. Song XJ, Wang ZB, Gan Q and Walters ET: cAMP and cGMP contribute to sensory neuron hyperexcitability and hyperalgesia in rats with dorsal root ganglia compression. J Neurophysiol 95: 479-492, 2006.

22. Bhatt DH, Otto SJ, Depoister B and Fetcho JR: Cyclic AMP-induced repair of zebrafish spinal circuits. Science 305: 254-258, 2004.

23. Nikulina E, Tidwell JL, Dai HN, Bregman BS and Filbin MT: The phosphodiesterase inhibitor rolipram delivered after a spinal cord lesion promotes axonal regeneration and functional recovery. Proc Natl Acad Sci USA 101: 8786-8790, 2004.

24. Pearse DD, Pereira FC, Marcillo AE, et al: cAMP and Schwann cells promote axonal growth and functional recovery after spinal cord injury. Nat Med 10: 610-616, 2004.

25. Page CP and Spina D: Selective PDE inhibitors as novel treatments for respiratory diseases. Curr Opin Pharmacol 12: 275-286, 2012.

26. Zhu J, Yang Q, Dai D and Huang Q: X-ray crystal structure of phosphodiesterase 2 in complex with a highly selective, nanomolar inhibitor reveals a binding-induced pocket important for selectivity. J Am Chem Soc 135: 11708-11711, 2013.

27. Liu S, Li Y, Kim S, et al: Phosphodiesterases coordinate cAMP propagation induced by two stimulatory $G$ protein-coupled receptors in hearts. Proc Natl Acad Sci USA 109: 6578-6583, 2012.

28. Perez-Torres S, Miro X, Palacios JM, et al: Phosphodiesterase type 4 isozymes expression in human brain examined by in situ hybridization histochemistry and $[3 \mathrm{H}]$ rolipram binding autoradiography. Comparison with monkey and rat brain. J Chem Neuroanat 20: 349-374, 2000

29. Lamontagne S, Meadows E, Luk P, et al: Localization of phosphodiesterase-4 isoforms in the medulla and nodose ganglion of the squirrel monkey. Brain Res 920: 84-96, 2001.

30. Abaffy T, Trubey KR and Chaudhari N: Adenylyl cyclase expression and modulation of cAMP in rat taste cells. Am J Physiol Cell Physiol 284: C1420-C1428, 2003.

31. Gavaldà A and Roberts RS: Phosphodiesterase-4 inhibitors: a review of current developments (2010-2012). Expert Opin Ther Pat 23: 997-1016, 2013.

32. Wu D, Zhao Y, Yang Y, Wang W and Xu R: A multicenter clinical study on clinical effects of meglumine cyclic adenylate in treating patients with chronic pulmonary heart disease]. Zhonghua nei ke za zhi 40: 467-470, 2001 (In Chinese).

33. Xing SH, Gu SL and Meng QC: Meglumine cyclic adenylate pediatric dosage calculations. XuZhou Acta Academiae Medicinae 19: 370-372, 1999. 